\title{
Line profile variability and tidal flows in eccentric binaries
}

\author{
Gloria Koenigsberger $^{1}$, Edmundo Moreno ${ }^{2}$ and David M. Harrington ${ }^{3}$ \\ ${ }^{1}$ Instituto de Ciencias Físicas, Universidad Nacional Autónoma de México, \\ Cuernavaca, Morelos, 62210, Mexico \\ email: gloria@astro.unam.mx \\ ${ }^{2}$ Instituto de Astronomía, Universidad Nacional Autónoma de México, \\ Apdo. Postal 70-264, México D.F. 04510, Mexico \\ email: edmundo@astro. unam.mx \\ ${ }^{3}$ Institute for Astronomy, University of Hawaii, \\ Box 2680 Woodlawn Drive, Honolulu, HI, 96822, USA \\ email: dmh@ifa.hawaii.edu
}

\begin{abstract}
A number of binary systems display enhanced activity around periastron passage which may be caused by the tidal interactions. We have developed a time-marching numerical calculation from first principles that computes the surface deformations, the perturbed velocity field, the energy dissipation rates and the photospheric line-profiles in a rotating star with a binary companion in an eccentric orbit. The method consists of solving the equations of motion for a grid of elements covering the surface of star $m_{1}$, subjected to gravitational, centrifugal, Coriolis, gas pressure and viscous shear forces (Moreno et al. 1999, Toledano et al. 2007, Moreno et al. 2011). At selected times during the orbital cycle, the velocities of surface elements on the visible hemisphere of the star are projected along the observer's line of sight and the photospheric line-profile calculation is performed (Moreno et al. 2005). Direct comparison with observational photospheric line profile variability is then possible, showing that the general features are reproduced (Harrington et al. 2009). In this poster we show the example of a highly eccentric system $(e=0.8, P=15 \mathrm{~d})$. The surface deformation changes rapidly from that of an "equilibrium tide" at periastron to one with smaller-scale structure shortly thereafter. The computed line profiles display the presence of large blue-to-red migrating "bumps" around periastron, with smaller scale structure appearing later in the orbital cycle. Because the growth rate of the surface perturbations increases very abruptly at periastron, instabilities are expected to arise which may cause the observed activity and mass-ejection events around this orbital phase.
\end{abstract}

Keywords. binaries: spectroscopic, stars: oscillations (including pulsations), stars: activity, line: profiles

\section{References}

Harrington, D., Koenigsberger, G., Moreno, E., \& Kuhn, J. 2009, ApJ, 704, 813

Moreno, E. \& Koenigsberger, G. 1999, Rev. Mexicana AyA, 35, 157

Moreno, E., Koenigsberger, G., \& Toledano, O. 2005, A\&SA, 437, 641

Moreno, E., Koenigsberger, G. \& Harrington, D. M. 2011, in preparation

Toledano, O., Moreno, E., Koenigsberger, G., \& Detmers, R. et al. 2007, A\&AA, 461, 1057 

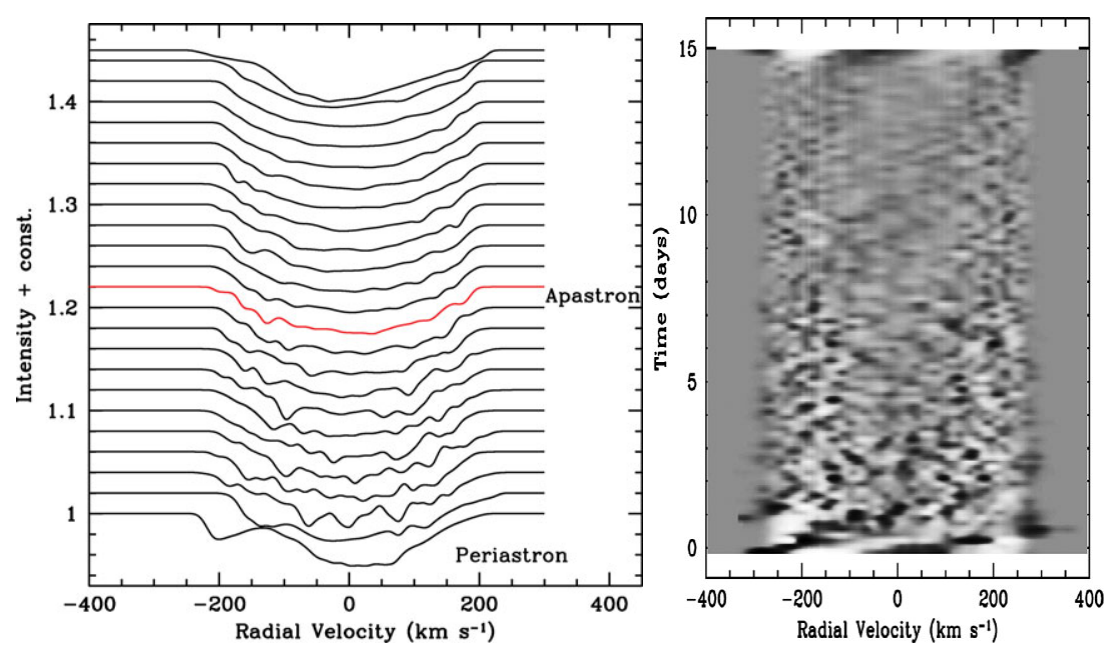

Figure 1. Example of computed photospheric line-profile variability arising in the primary star $\left(m_{1}=5 \mathrm{M}_{\odot}, R_{1}=3.2 \mathrm{R}_{\odot}\right)$ of a highly eccentric $(e=0.8), 15$-day binary system. The initial equatorial rotation velocity is $192 \mathrm{~km} \mathrm{~s}^{-1}$, which corresponds to a rotation rate which is 1.2 faster than the relative orbital motion of the companion at periastron. The companion's mass is $m_{2}=4 \mathrm{M}_{\odot}$. The left panel contains a sample of the computed line profiles, distributed evenly over the orbital cycle, starting with periastron passage at the bottom of the plot. Although "bumps" are visible throughout the orbital cycle, their amplitude is clearly largest around periastron. In the grey-scale representation of the line-profile residuals (right) the transition from broad blue-to-red migrating "bumps" at periastron to smaller-scale structure a little later in the orbital cycle is evident.

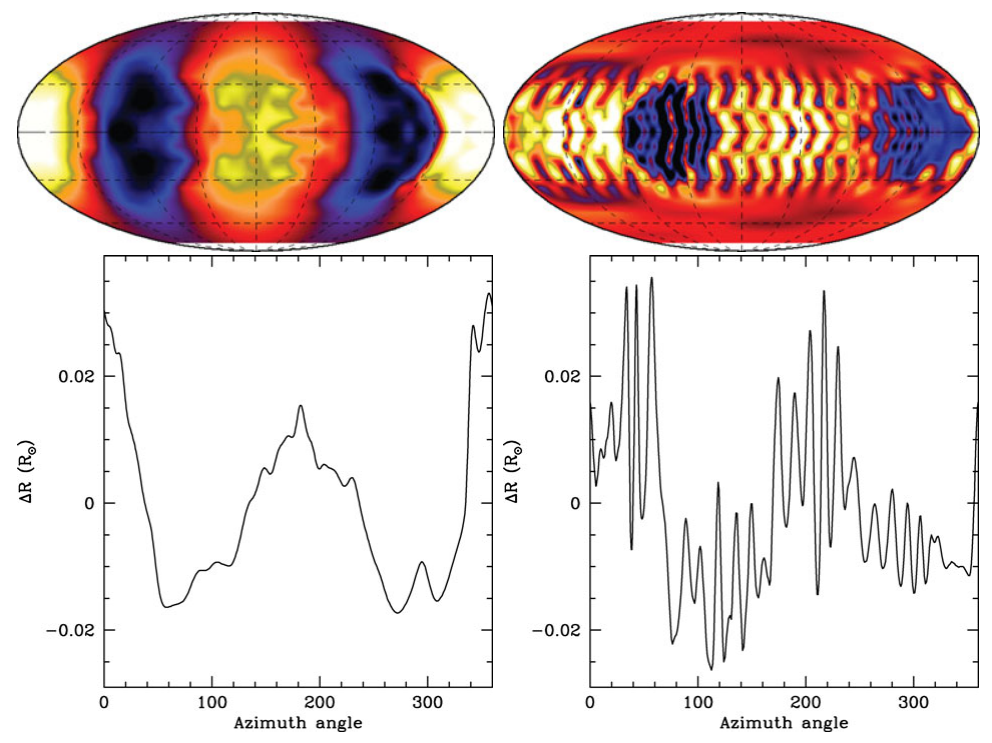

Figure 2. Example of the surface distortion at two orbital phases, periastron (left) and 0.833 days later (right) for the same model binary system described in Fig. 1. These figures illustrate the rapid change from an "equilibrium tide" configuration at periastron to one with structures of a much smaller scale. The maps (top) are in Mollweide projection, with azimuth angle $\varphi=0^{\circ}$ at left and $360^{\circ}$ at right. The companion, $m_{2}$, is located at $\varphi=0^{\circ}$. White corresponds to maximum positive deformation. The plots (bottom) give the amplitude in $R_{\odot}$ of the deformation at the equatorial latitude. 